\title{
Ventricular pacemaker upgrade: experience, complications and recommendations
}

\author{
D J R Hildick-Smith, M D Lowe, S A Newell, P M Schofield, L M Shapiro, D L Stone, \\ A A Grace, M C Petch
}

\begin{abstract}
Objective-To assess outcomes of pacemaker upgrade from single chamber ventricular to dual chamber.

Design-Retrospective analysis of patients undergoing the procedure.

Setting-Specialist cardiothoracic unit.

Patients-44 patients (15 female, 29 male), mean (SD) age at upgrade 68.2 (12.9) years.
\end{abstract}

Interventions-Upgrade of single chamber ventricular to dual chamber pacemaker.

Main outcome measures-Procedure duration and complications.

Results-Principal indications for upgrade were pacemaker syndrome (17), "opportunistic"-that is, at elective generator replacement (8), heart failure (7), non-specific breathlessness/fatigue (7), and neurally mediated syncope (3). Mean (SD) upgrade procedure duration (82.9 (32.6) minutes) significantly exceeded mean VVI implantation duration (42.9 (13.3) minutes) and mean DDD implantation duration (56.6 (22.7) minutes) (both p < 0.01). Complications included pneumothorax (1), ventricular arrhythmia requiring cardioversion (2), protracted procedure (10), atrial lead repositioning within six weeks (8), haematoma evacuation (1), superficial infection (1), and admission to hospital with chest pain (1); 20 patients $(45 \%)$ suffered one or more complications including four of the eight who underwent opportunistic upgrade.

Conclusions-Pacemaker upgrade takes longer and has a higher complication rate than either single or dual chamber pacemaker implantation. This suggests that the procedure should be performed by an experienced operator, and should be undertaken only if a firm indication exists. Patients with atrial activity should not be offered single chamber ventricular systems in the belief that the unit can be upgraded later if necessary at minimal risk.

(Heart 1998;79:383-387)

Keywords: pacemaker upgrade; dual chamber pacemaker; single chamber pacemaker

Since the introduction of the dual chamber pacemaker, "upgrading" of single chamber ventricular pacemakers to dual chamber units has been a possibility in patients with persisting atrial electrical activity. Current guidelines suggest that patients with intrinsic atrial activity should receive dual chamber implants (or single chamber atrial implants if appropriate), ${ }^{23}$ but in the United Kingdom $40 \%$ of patients paced for high grade atrioventricular block during 1995 received fixed rate ventricular pacing systems (National Pacemaker Database, 1996) for reasons that were likely to include age, ${ }^{45}$ cost,${ }^{6}$ relative inactivity, ${ }^{4}$ and intermittency of atrioventricular block. ${ }^{7}$ Patients receiving such relatively simple systems lack atrioventricular synchrony and a proportion (variously estimated at between $15 \%$ and $70 \%^{8-11}$ ) go on to experience symptoms of the pacemaker syndrome..$^{12}$ These patients, and those whose symptoms persist for other reasons (such as heart failure), may be considered for pacemaker upgrade.

There has only been one study concerning upgrading of pacemakers. This was undertaken in an asymptomatic population who underwent opportunistic upgrade at the time of elective generator change. ${ }^{11}$ There are no data relating to clinical experience of pacemaker upgrade in other patients. We sought to address this issue in a cardiology centre with a large pacemaker practice, assessing procedural characteristics, complication rates, and symptom response.

\section{Methods}

In this specialist cardiothoracic unit, approximately 350 permanent pacing systems are implanted annually. In the period under review (June 1989 to June 1997), 2614 patients received permanent pacemakers: 794 (31\%) dual chamber, $1820(69 \%)$ single chamber. Of those who received single chamber units, 986 were paced for high grade atrioventricular block (628) or sinus node disease (358), of whom $44(4.5 \%)$ have subsequently undergone upgrading of single chamber ventricular units to dual chamber units. These patients formed the study group. Information regarding indications for upgrade, procedure duration, complications, symptom response, and subsequent clinical course were analysed. Complications of the pacing procedure were taken to include protracted upgrade procedure (defined as mean dual chamber implantation time plus twice the standard deviation from a random sample of 100 age matched implants, that is, $>102$ minutes), recognised procedural adverse events (for example, pneumothorax, arrhythmia requiring cardioversion), pacemaker reexploration within six weeks, or other hospital admission as a result of upgrade. These outcomes were recorded, and symptom response to upgrade was graded subjectively according to a three point scale. 
Table 1 Original indication for pacing $(n=44)$

\begin{tabular}{lc}
\hline Indication & No of patients \\
\hline Complete heart block & 17 \\
Sinus node disease & 8 \\
Second degree atrioventricular block & 7 \\
Paroxysmal atrial fibrillation with pauses in & \\
$\quad$ ventricular response & 3 \\
Trifascicular block & 3 \\
Neurally mediated syncope & 3 \\
Bifascicular block with syncope & 2 \\
Hypertrophic cardiomyopathy & 1 \\
\hline
\end{tabular}

DATA ANALYSIS

Data distribution was assessed for normality. Data are expressed as mean (SD), or median (SEM), depending on distribution. Comparison of procedure duration was made with the Mann-Whitney U test, or the median test as appropriate. Comparison between procedure outcomes or characteristics was made with the $\chi^{2}$ test or Fisher's exact test as appropriate. The null hypothesis was rejected at the level $\mathrm{p}<0.05$.

\section{Results}

Between June 1989 and June 1997, 44 patients underwent attempted upgrade of a single chamber ventricular pacing system to a dual chamber system. Fifteen $(36 \%)$ were female and $29(64 \%)$ male. Average age at the time of original implant was 62.3 (13.8) years. Indications for original implant are shown in table 1.

Thirty three patients benefited symptomatically following original implant. Six required re-exploration of their original pacing system, for haematoma evacuation (1), ventricular lead repositioning (2), infection (1), lead erosion (1), and persistent syncope (1). Three patients underwent elective generator replacement in the interval between initial implant and upgrade.

The interval between original implant and upgrade was 5.9 (4.5) years, and the average age at upgrade was 68.2 (12.9) years. Indications for upgrade and symptom response to the procedure are shown in table 2 . Of the 44 attempted upgrade procedures, 43 were successfully completed.

PROCEDURE DURATION

Upgrade procedure duration (82.9 (32.6) minutes) significantly exceeded single chamber pacemaker implantation (42.9 (13.3) minutes, $\mathrm{p}<0.01$ ) or dual chamber pacemaker implantation (56.6 (22.7) minutes, $\mathrm{p}<0.01$ ), both assessed from 100 randomly selected age matched procedures (table 3 ).

Table 2 Indication for upgrade of pacing unit and symptomatic response to upgrade

\begin{tabular}{|c|c|c|c|c|}
\hline \multirow[b]{2}{*}{ Indication } & \multirow{2}{*}{$\begin{array}{l}\text { No of patients } \\
(n=44)\end{array}$} & \multicolumn{3}{|c|}{$\begin{array}{l}\text { Symptom resolution following } \\
\text { upgrade }\end{array}$} \\
\hline & & Complete & Partial & None \\
\hline Pacemaker syndrome & 17 & 11 & 4 & 2 \\
\hline Opportunistic (at generator change) & 8 & \multicolumn{3}{|c|}{ Not applicable ${ }^{\star}$} \\
\hline Symptoms of heart failure & 7 & 0 & 3 & $4 \dagger$ \\
\hline Non-specific breathlessness or fatigue & 7 & 0 & 4 & 3 \\
\hline Neurally mediated syncope & 3 & 2 & 1 & 0 \\
\hline Progression to complete heart block & 1 & 1 & 0 & 0 \\
\hline \multicolumn{5}{|l|}{ Prevention of paroxysms of atrial } \\
\hline fibrillation & 1 & 0 & 0 & 1 \\
\hline
\end{tabular}

${ }^{\star}$ One patient noted significant improvement in overall wellbeing.

tIncludes one patient in whom the upgrade procedure could not be completed.
Table 3 Procedure duration

\begin{tabular}{lll}
\hline Procedure & $\begin{array}{l}\text { No of } \\
\text { patients }\end{array}$ & $\begin{array}{l}\text { Mean (SD) } \\
\text { time (minutes) }\end{array}$ \\
\hline $\begin{array}{l}\text { Single chamber pacemaker } \\
\quad \text { implantation }\end{array}$ & 100 & $42.9(13.3)^{\star}$ \\
$\begin{array}{l}\text { Dual chamber pacemaker } \\
\text { implantation }\end{array}$ & 100 & $56.6(22.7)$ \\
$\quad$ Ventricular pacemaker upgrade & 44 & $82.9(32.6) \dagger \ddagger$ \\
\hline
\end{tabular}

$\star$ Single $v$ dual $\mathrm{p}<0.05 ;$ tsingle $v$ upgrade $\mathrm{p}<0.001 ; \ddagger$ dual $v$ upgrade $\mathrm{p}<0.001$.

COMPLICATIONS

Ten patients underwent protracted procedures (defined above, > 102 minutes) Reasons included difficult dissection (3), adaptation of atrial or ventricular lead (2), difficulty establishing venous access (1), repeated atrial displacement (1), persistent left sided superior vena cava (1), difficulty in establishing adequate atrial parameters (1), and inability to sense or pace atrium (1).

Procedural adverse events were encountered in three patients. These were ventricular arrhythmia requiring DC cardioversion (2) and pneumothorax (1). Nine patients $(20 \%)$ underwent re-exploration of their pacing system within six weeks of the upgrade. Of these, eight were for atrial lead displacement (seven within 24 hours) and one for haematoma evacuation. Two patients were readmitted to hospital in the postoperative period for symptoms related to the upgrade procedure, one for superficial infection over the pacing generator (on three separate occasions), and one for chest pain. In total, 20 patients $(45 \%)$ experienced one or more complications (fig 1). Median hospital stay was two nights, but eight patients $(18 \%)$ remained in hospital for one or more extra nights as a result of complications.

UPGRADE AT ELECTIVE UNIT REPLACEMENT

Eight patients underwent "opportunistic" upgrade only, in that their pacing system was upgraded at the time of elective generator replacement despite the fact that they were asymptomatic in VVI mode. Of these patients, three had protracted procedures, one developing superficial infection requiring subsequent hospital admission. One further patient underwent repositioning of the atrial lead at two weeks. Therefore four of eight patients who underwent opportunistic upgrade of their pacing system encountered complications.

\section{PROCEDURAL CHARACTERISTICS}

The original implant was via the cephalic vein in seven patients and the subclavian vein in the remaining 37. Upgrade approaches were cephalic (4) and subclavian (40). In 11 patients the approach used for the upgrade was different from that used for the original implant; procedure duration (median (SEM)) did not differ according to whether the venous approach employed for the upgrade was different or the same $(70(12.6) v 80$ (5.7) minutes, respectively, $\mathrm{p}=0.21$ ), nor did complication rates differ significantly.

The atrial lead initially chosen for use was passive fixation in 32 patients and active in 12 . Of these, three passive atrial leads failed to 


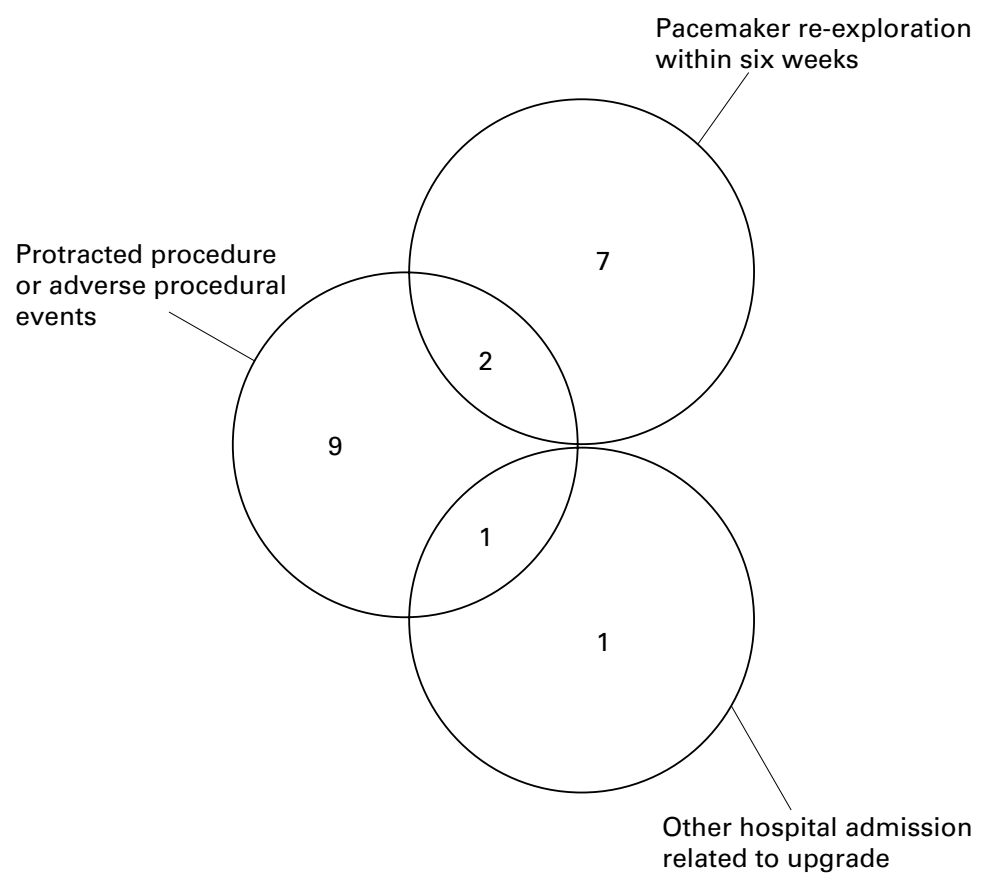

Figure 1 Complications of 44 upgrade procedures.

establish adequate atrial parameters or stability, and were exchanged for active leads, two successfully (one in a patient with persistent left superior vena cava), one unsuccessfully (in a patient with dystrophia myotonica). Complications were encountered in four of 12 active fixation procedures and 16 of 32 passive fixation procedures (NS).

INFLUENCE OF OPERATOR EXPERIENCE

Eighteen operators undertook pacemaker upgrade, performing between one and six procedures each. Thirteen procedures were undertaken by relatively inexperienced operators ( $<100$ permanent pacing procedures) and 31 by experienced operators ( $>100$ procedures). Procedure duration (median (SEM)) was, respectively, 85 (7.8) $v 75$ (5.5) minutes $(\mathrm{p}=0.22$, NS). Protracted procedure, operative complications, re-exploration, or other hospital admission occurred in seven (of 13) inexperienced operator procedures $v 13$ (of 31) experienced operator procedures $(\mathrm{p}=0.45$, NS). Five operators performed more than two upgrade procedures, and 17 procedures were undertaken by operators with experience of more than two previous procedures. Procedure duration for these "experienced upgrader" cases (median (SEM)) was 75 (8.5) minutes, compared with 75 (5.2) minutes for "inexperienced upgrader" procedures $(p=0.92, \mathrm{NS})$. Complications, however, occurred in only four of 17 experienced upgrader cases $(24 \%)$ compared with 16 of 27 inexperienced upgrader cases $(59 \% ; \mathrm{p}=0.022)$. Complication rates were therefore significantly reduced after an operator's second upgrade procedure.

FOLLOW UP

Two patients subsequently underwent late pacing system revision, 15 and 48 months after upgrade respectively, both for ventricular lead failure. The upgrade procedure was not directly implicated in either case, though this possibility cannot be discounted. Seven patients have subsequently died, all of whom had poor left ventricular function at the time of upgrade, and four of whom underwent upgrade in an attempt to improve symptoms of heart failure. The interval between upgrade and death was $2,4,5,6,10,28$, and 30 months, respectively. All deaths were caused by heart failure, and none was attributable to the pacing procedure.

\section{Discussion}

Many patients undergo single chamber ventricular pacemaker implantation despite the presence of organised atrial electrical activity (UK National Pacemaker Database, 1996). Some of these patients later require system upgrade as a result of pacemaker syndrome or unresolved symptoms. Pacing upgrade is a more complex procedure than initial system implantation, but there are no studies assessing procedural characteristics and complications of this procedure. Sulke et al took 16 patients asymptomatic in VVI mode and upgraded their pacing systems at elective generator replacement. ${ }^{11}$ These patients experienced an improvement in perceived general wellbeing, and an increase in exercise capacity in DDD mode, but complications of the upgrade procedure which might have offset this potential benefit were not discussed. Other studies have compared VVI, DDI, and DDD modes, but these studies are confined to patients with dual chamber pacing systems already in situ. ${ }^{13-15}$

The most striking findings of our study concern procedure duration and early complications. Pacemaker upgrade took, on average, twice as long as single chamber ventricular implant, and almost half an hour longer than dual chamber implant, illustrating the technically more challenging nature of the procedure. Dissection of fibrosed and adherent tissue planes, attainment of venous access avoiding damage to the existing lead, and adaptation of ventricular or atrial leads to fit a new or old generator may all present difficulties. Ten procedures in our series were protracted, and five took over two hours, thereby increasing patient discomfort and risk of infection (seen in one of our patients).

Atrial lead displacement occurring within six weeks of upgrade was seen in eight patients (18\%), compared with 11 of 286 patients who underwent dual chamber implants at this centre between 1987 and 1993 (3.8\%), ${ }^{16}$ and with a standard atrial displacement rate of $1 \%{ }^{17}$ to $5 \%{ }^{7}$ The reason for the high incidence of atrial displacement at upgrade is not immediately apparent. Contributory factors may include difficulties in manipulating the atrial lead through inadequately dissected access, relatively superficial lead suturing, and inadvertent traction during lead adaptation or lead insertion into the generator or pocket. Patients with heart failure may have large myopathic atria with poor sensing and pacing characteristics, and poor lead stability. There were several such patients in our series. Relative operator inexperience is also a factor. Protracted upgrade 
procedure, procedural complications, pacemaker re-exploration within six weeks, or other hospital admission as a result of upgrade were seen more commonly, though not to a level of statistical significance, among relatively inexperienced operators ( $<100$ procedures). Complications were significantly more common, however, when comparing operators inexperienced with those experienced at upgrade procedures. Complications were statistically reduced after an operator's second upgrade procedure, consistent with a steep but important learning curve.

Greatest symptomatic benefit was seen in those who underwent upgrade for pacemaker syndrome, neurally mediated syncope (with cardioinhibitory component), or development of complete heart block, as would be expected. ${ }^{13-15} 1819$ Seven patients underwent pacemaker upgrade in an attempt to improve symptoms of heart failure, as DDD pacing in these patients has been shown to be of potential benefit. $^{20-22}$ Results were relatively disappointing, in that four derived no symptomatic benefit while three benefited to some extent. Least clinical improvement was seen in those who underwent upgrade for non-specific breathlessness or fatigue in the absence of objective evidence of heart failure.

"Opportunistic" upgrading of a single chamber ventricular pacemaker to a dual chamber unit at the time of elective unit replacement remains contentious. Morbidity and mortality are greater in patients with persisting atrial activity paced in VVI mode than in modes which preserve atrioventricular synchrony, ${ }^{23-29}$ but this does not necessarily mean that these patients will benefit from an upgrade procedure with its attendant risks. Sulke et al showed that patients in asymptomatic complete heart block can benefit from upgrade, ${ }^{11}$ and therefore suggested the concept of "subclinical" pacemaker syndrome, but potential complications of the procedure itself were not taken into account. In our series, eight patients underwent opportunistic upgrade, four of whom encountered problems. The potential benefit of upgrading in our asymptomatic subjects was therefore more than offset by the increased morbidity associated with the procedure itself. We suggest that pacemaker upgrade is not usually warranted in asymptomatic individuals, and should only be undertaken after careful consideration, by an operator experienced in upgrade procedures.

Pacemaker upgrade may be carried out with active or passive fixation atrial electrodes. It may be prudent to consider active fixation if the patient has previously undergone cardiac surgery, ${ }^{30}$ but this precaution is not always necessary, and may make use of the cephalic approach awkward. We found no significant difference in complication rate between passive and active atrial lead selection.

Mode of venous access for upgrade did not significantly influence complication rate, but the four patients who underwent cephalic upgrade following subclavian implant experienced no complications. The cephalic upgrade approach minimises risk of damage to the existing pacing lead, and removes risk of pneumothorax. We suggest that if the original implant was by subclavian vein puncture then upgrade should be undertaken through the cephalic vein.

The UKPACE trial will compare VVI, VVIR, and DDD pacing modes in elderly patients (over 70 years) with high grade atrioventricular block, and should provide clarification of the benefits (or otherwise) of sophisticated pacing in such patients. However, pending the results of this trial, the incidence of complications at upgrade that we have shown should mitigate against the use of VVI pacing in patients with ongoing atrial activity, a practice which remains commonplace, ${ }^{31}$ despite the fact that the true incidence of pacemaker syndrome in such patients may exceed $50 \% .^{81132}$ If expertise and budgets permit, these patients should be offered dual chamber units at the outset, rather than risk the need for upgrade at a later date.

CONCLUSIONS

Pacemaker upgrade is technically challenging and requires particular expertise. It should not be undertaken in asymptomatic subjects without considerable thought, and should be performed by an experienced operator. Pacemaker upgrade takes longer and has a higher complication rate than either single or dual chamber pacemaker implantation. Patients with atrial activity should not be offered single chamber ventricular systems on the pretext that the unit can be upgraded if necessary at minimal risk.

1 Parsonnet V. Routine implantation of permanent transvenous pacemaker electrodes in both chambers. A technique whose time has come. Pacing Clin Electrophysiol 1981;4:109-12

2 Clarke M, Sutton R, Ward D, et al. Recommendations for pacemaker prescription for symptomatic bradycardia. Report of a working party of the British Pacing and Report of a working party of the British Pacing
Electrophysiology Group. Br Heart $\mathcal{F}$ 1991;66:185-91.

3 Dreifus LS, Fisch C, Griffin JC, et al. Guidelines for implantation of cardiac pacemakers and antiarrhythmia devices. A report of the American College of Cardiology/ American Heart Association Task Force on Assessment of American Heart Association Task Force on Assessment of
Diagnostic and Therapeutic Cardiovascular Procedures Committee on Pacemaker Implantation). Circulation $1991 ; 84: 455-67$

4 Lamas GA, Pashos CL, Normand SL, et al. Permanent pacemaker selection and subsequent survival in elderly Medicare pacemaker recipients. Circulation 1995;91:10639.

5 Aggarwal RK, Ray SG, Connelly DT, et al. Trends in pacemaker mode prescription 1984-1994: a single centre study of 3710 patients. Heart 1996;75:518-21.

6 de Belder MA, Linker NJ, Jones S, et al. Cost implications of the British Pacing and Electrophysiology Group's recommendations for pacing. BMF 1992;305:861-5.

7 Goldman BS, Fraser JD, Morgan CD. Survey of cardiac pacing in Canada. Can f Cardiol 1991;7:391-8.

8 Heldman D, Mulvihill D, Nguyen H, et al. True incidence of pacemaker syndrome. Pacing Clin Electrophysiol 1990;13: $1742-50$.

9 Furman S. Pacemaker syndrome. Pacing Clin Electrophysiol 1994;17:1-5.

10 Ausubel K, Furman S. The pacemaker syndrome. Ann Intern Med 1985;103:420-9.

11 Sulke N, Dritsas A, Bostock J, et al. "Subclinical” pacemaker syndrome: a randomised study of symptom free patients with ventricular demand (VVI) pacemakers upgraded to dual chamber devices. Br Heart f 1992;67:57-64.

12 Levine PA, Seltzer JP, Pirzada FA. The "pacemaker syndrome" in a properly functioning physiologic pacing system. Pacing Clin Electrophysiol 1983;6:279-82.

13 Hargreaves MR, Channon KM, Cripps TR, et al. Comparison of dual chamber and ventricular rate responsive pacing in patients over 75 with complete heart block. Br Heart $\mathcal{f}$ in patients over 75

14 Sulke N, Chambers J, Dritsas A, et al. A randomized double-blind crossover comparison of four rate-responsive pacing modes. F Am Coll Cardiol 1991;17:696-706. 
15 Channon KM, Hargreaves MR, Cripps TR, et al. DDD vs. VVI pacing in patients aged over 75 years with complete ele-blind crossover comparison. $Q \mathcal{7} M e d$ 1994;87:245-51

16 Chauhan A, Grace AA, Newell SA, et al. Early complications after dual chamber versus single chamber pacemake implantation. Pacing Clin Electrophysiol 1994;17:2012-15.
7 Mueller X, Sadeghi H, Kappenberger L. Complications

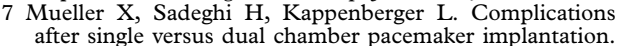
after single versus dual chamber pacema
Pacing Clin Electrophysiol 1990;13:711-14.

18 Petersen ME, Sutton R. Cardiac pacing for vasovagal syncope: a reasonable therapeutic option? Pacing Clin Electrophysiol 1997;20:824-6.

19 Luk1 J, Doupal V, Heinc P. Quality-of-life during DDD and dual sensor VVIR pacing. Pacing Clin Electrophysiol 1994;17:1844-8.

20 Hochleitner $\mathrm{M}$, Hortnagl $\mathrm{H}, \mathrm{Ng} \mathrm{CK}$, et al. Usefulness of physiologic dual-chamber pacing in drug-resistant idiopathic dilated cardiomyopathy. Am f Cardiol 1990;66: opathic 1982 .

21 Auricchio A, Sommariva L, Salo RW, et al. Improvement of cardiac function in patients with severe congestive heart failure and coronary artery disease by dual chamber pacing with shortened AV delay. Pacing Clin Electrophysiol 1993;16: 2034-43.

22 Hochleitner M, Hortnagl H, Hortnagl H, et al. Long-term efficacy of physiologic dual-chamber pacing in the treatment of end-stage idiopathic dilated cardiomyopathy. Am F Cardiol 1992;70:1320-5.

23 Santini M, Alexidou G, Ansalone G, et al. Relation of prognosis in sick sinus syndrome to age, conduction defects and modes of permanent cardiac pacing. Am f Cardiol 1990;65: 729-35.
24 Rosenqvist $\mathrm{M}$, Brandt J, Schuller $\mathrm{H}$. Long-term pacing in sinus node disease: effects of stimulation mode on cardiovascular morbidity and mortality. Am Heart $\mathcal{f}$ 1988;116:16-22.

25 Andersen HR, Thuesen L, Bagger JP, et al. Prospective randomised trial of atrial versus ventricular pacing in sick-sinus syndrome. Lancet 1994;344:1523-8.

26 Sgarbossa EB, Pinski SL, Maloney JD, et al. Chronic atrial fibrillation and stroke in paced patients with sick sinus syndrome. Relevance of clinical characteristics and pacing modalities. Circulation 1993;88:1045-53.

27 Hesselson AB, Parsonnet V, Bernstein AD, et al. Deleterious effects of long-term single-chamber ventricular pacing in patients with sick sinus syndrome: the hidden benefits of dual-chamber pacing. 7 Am Coll Cardiol 1992;19:1542-9.

28 Nielsen JC, Pedersen AK, Mortensen PT, et al. Atrial versus ventricular pacing in patients with sick sinus syndrome. Long-term follow-up in a prospective, randomized trial of 225 consecutive patients [abstract]. Eur Heart f 1997;18: 482.

29 Andersen HR, Nielsen JC, Thomsen PE, et al. Long-term follow-up of patients from a randomised trial of atrial versus ventricular pacing for sick sinus syndrome. Lancet 1997;350:1210-16.

30 Masterson MM, Maloney JD, Tuzcu EM, et al. Atrial pacemaker leads compared. Cleve Clin f Med 1990;57:433-6.

31 Toff W, Skehan J, De Bono D, et al. The United Kingdom pacing and cardiovascular events (UKPACE) trial. Heart 1997;78:221-3.

32 Travill CM, Sutton R. Pacemaker syndrome: an iatrogenic condition. Br Heart $\mathcal{F}$ 1992;68:163-6. 